\title{
Spectrally Switchable Photodetection with Near-Infrared-Absorbing Covalent Organic Frameworks
}

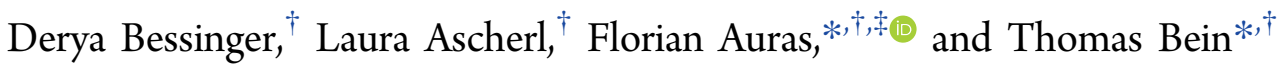 \\ ${ }^{\dagger}$ Department of Chemistry and Center for NanoScience (CeNS), University of Munich (LMU), Butenandtstraße 5-13, 81377 \\ Munich, Germany \\ ${ }^{\ddagger}$ Cavendish Laboratory, University of Cambridge, Cambridge CB3 0HE, United Kingdom
}

Supporting Information

\begin{abstract}
Most covalent organic frameworks (COFs) to date are made from relatively small aromatic subunits, which can only absorb the high-energy part of the visible spectrum. We have developed near-infrared-absorbing low bandgap COFs by incorporating donor-acceptor-type isoindigo- and thienoisoindigo-based building blocks. The new materials are intensely colored solids with a high degree of long-range order and a pseudo-quadratic pore geometry. Growing the COF as a vertically oriented thin film allows for the construction of an ordered interdigitated heterojunction through infiltration with a complementary semiconductor. Applying a thienoisoindigo-COF:fullerene heterojunction as the photoactive component, we realized the first COF-based UV- to NIR-responsive

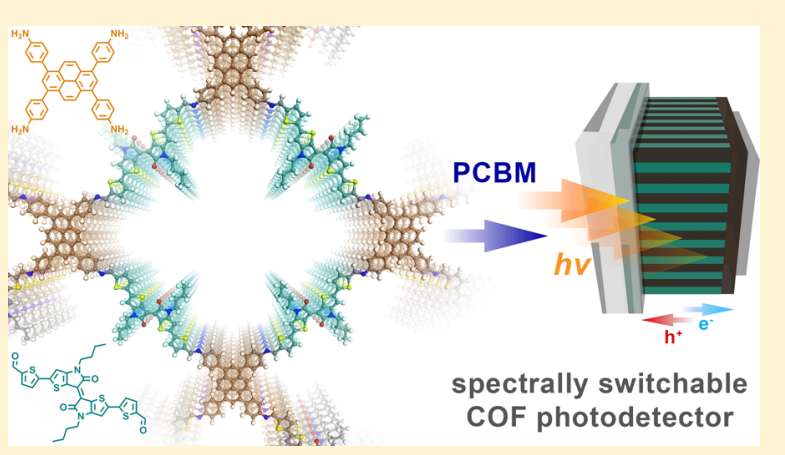
photodetector. We found that the spectral response of the device is reversibly switchable between blue- and red-sensitive, and green- and NIR-responsive. To the best of our knowledge, this is the first time that such nearly complete inversion of spectral sensitivity of a photodetector has been achieved. This effect could lead to potential applications in information technology or spectral imaging.
\end{abstract}

\section{INTRODUCTION}

Constructing covalent organic frameworks (COFs) from rigid organic building blocks has opened a synthetic route to a broad range of tailor-made porous materials, ${ }^{1-3}$ whereby key properties such as the pore structure, optical properties and electronic coupling between the individual subunits can be tuned via the selection of suitable building blocks. ${ }^{4-6}$ The materials realized this way offer a unique combination of high thermal and mechanical stability, ${ }^{7}$ very high surface areas, ${ }^{8,9}$ and high density and accessibility of the functional organic building blocks, ${ }^{10}$ thus rendering them suitable for potential applications in gas storage and separation, ${ }^{11}$ catalysis and photocatalysis, ${ }^{12,13}$ and optoelectronics. ${ }^{14-16}$

The geometry of stacked two-dimensional (2D) COFs can be designed to tune the $\pi$-overlap between the individual layers, ${ }^{17,18}$ thus creating conductive columns and/or facilitating long-range exciton transport. ${ }^{19-21}$ Initial studies have shown that COFs can act as the active component in interdigitated heterojunction photovoltaic devices. ${ }^{22-24}$ Due to the limited absorption capabilities of the building blocks used in these studies, however, the devices could only harvest light in the blue and green spectral regions with appreciable quantum efficiency. Extension of the absorption capabilities into the red and near-infrared (NIR) spectral regions that encompass most of the solar photons would therefore be highly desirable.

Since the maximum length of COF building blocks to date is limited to only $1-2 \mathrm{~nm}$ due to the increasing flexibility of more extended molecules, shifting the building block absorption into the NIR cannot be achieved by simply extending the length of the $\pi$-conjugated backbone. However, combining electron-rich and -deficient moieties within the same building block can lead to additional charge-transfer transitions at energies well below the fundamental $\pi-\pi^{*}$ transition. ${ }^{25}$

One of the most effective components in this context is the highly electron-deficient isoindigo (II), an isomer of the naturally occurring indigo dye. Pairing isoindigo with an appropriate electron-rich counterpart leads to a strong intramolecular donor-acceptor charge delocalization in conjugated polymeric semiconductors, hence shifting the absorption onset well into the NIR regime. ${ }^{26}$ Bulk heterojunction solar cells with isoindigo-based low-band gap copolymers recently exceeded a power conversion efficiency of $9 \%$ with very high short-circuit currents of above $17 \mathrm{~mA} \mathrm{~cm}{ }^{-2}$. $^{27}$ The planarity and the highly polar nature of the isoindigo unit facilitate aggregation and give rise to exceptionally crystalline polymers, leading to high charge carrier mobilities and thus rendering isoindigo-containing polymers interesting candidates for organic field-effect transistor applications. ${ }^{28,29}$ Given these excellent electronic and optoelectronic properties, it would be highly desirable to be able to incorporate and study isoindigo-

Received: June 25, 2017

Published: August 22, 2017 

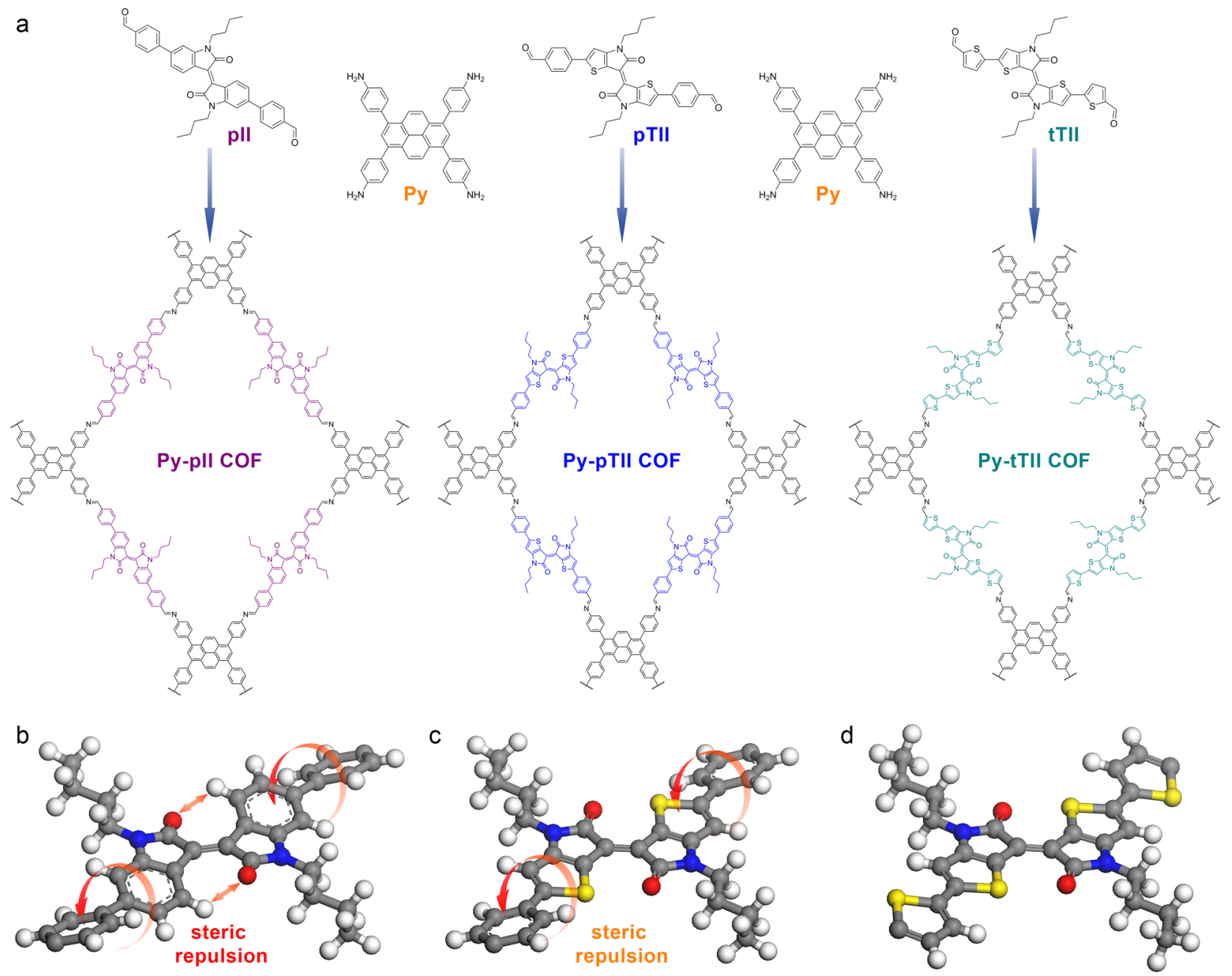

Figure 1. (a) Co-condensation of 1,3,6,8-tetrakis(4-aminophenyl)pyrene (Py) with 2 equiv of the (thieno-)isoindigo-bridged dialdehydes pII, pTII, and tTII leads to the formation of the respective imine-linked 2D COFs. (b) The cut-out of the simulated Py-pII COF structure reveals that the pII building block is not entirely flat. Steric repulsion causes a rotation of the terminal phenyls vs the isoindigo core. Additionally, the repulsion between the ketones and adjacent hydrogen atoms leads to a slight distortion of the core itself. (c) These steric constraints are considerably relaxed when the benzene rings are replaced by thiophenes, resulting in a planar thienoisoindigo core. While the terminal phenyl rings remain slightly rotated vs the core in the case of pTII, exchanging them for thiophenes (d) yields the virtually planar tTII building block.

based building blocks in the precisely defined environment of a $\mathrm{COF}^{30}$

In this work, we have developed a series of isoindigo- and thienoisoindigo-based building blocks and have applied them in the synthesis of highly crystalline imine-linked 2D COFs. The resulting materials are porous and intensely colored solids that absorb light throughout the visible and NIR spectral region. Growing vertically oriented thin films of these materials allows for the subsequent infiltration of the pores with a complementary semiconductor. The resulting ordered interdigitated heterojunction was employed as the active layer in the first COF-based NIR-sensitive and voltage-switchable photodetector.

\section{RESULTS AND DISCUSSION}

To create an isoindigo-bridged COF, we designed a new 6,6'bis(4-formylphenyl)- $N, N^{\prime}$-dibutyl-isoindigo (pII) building block. The terminal aldehyde hereby provides the chemical functionality for reversible imine formation, while the butyl chains ensure sufficient solubility during COF synthesis (Figure 1a).
In solution, the optical absorption spectrum of the pII building block exhibits two main absorption bands that are typical for donor-acceptor molecules (Supporting Information (SI), Figure S5a). The band at 370-470 $\mathrm{nm}$ has been attributed to the isoindigo $\pi-\pi^{*}$ transitions, whereas the lowerenergy band between 470 and $620 \mathrm{~nm}$ is due to an intramolecular charge transfer (ICT) between the electrondeficient isoindigo moiety and the more electron-rich phenylenes. ${ }^{31}$ The optical band gap, estimated from the corresponding Tauc plot for a direct allowed transition, is $2.06 \mathrm{eV}$ (SI, Figure $\mathrm{S} 5 \mathrm{~b})$.

Given the geometry of the building block (Figure 1b) and the strong tendency of isoindigo-based molecules to form closely packed, slightly offset cofacial aggregates, ${ }^{32}$ the construction of a geometrically compatible framework is crucial for obtaining a highly crystalline COF. Force-field- and density functional theory (DFT)-based simulations indicate that a synchronized slip-stacked arrangement in a pseudo-quadratic network, as induced by 1,3,6,8-tetrakis(4-aminophenyl)pyrene (Py), could match the geometric requirements of the pII unit very well. ${ }^{17}$ Among the known multidentate amine building blocks, Py has proven to produce some of the closest-packed 

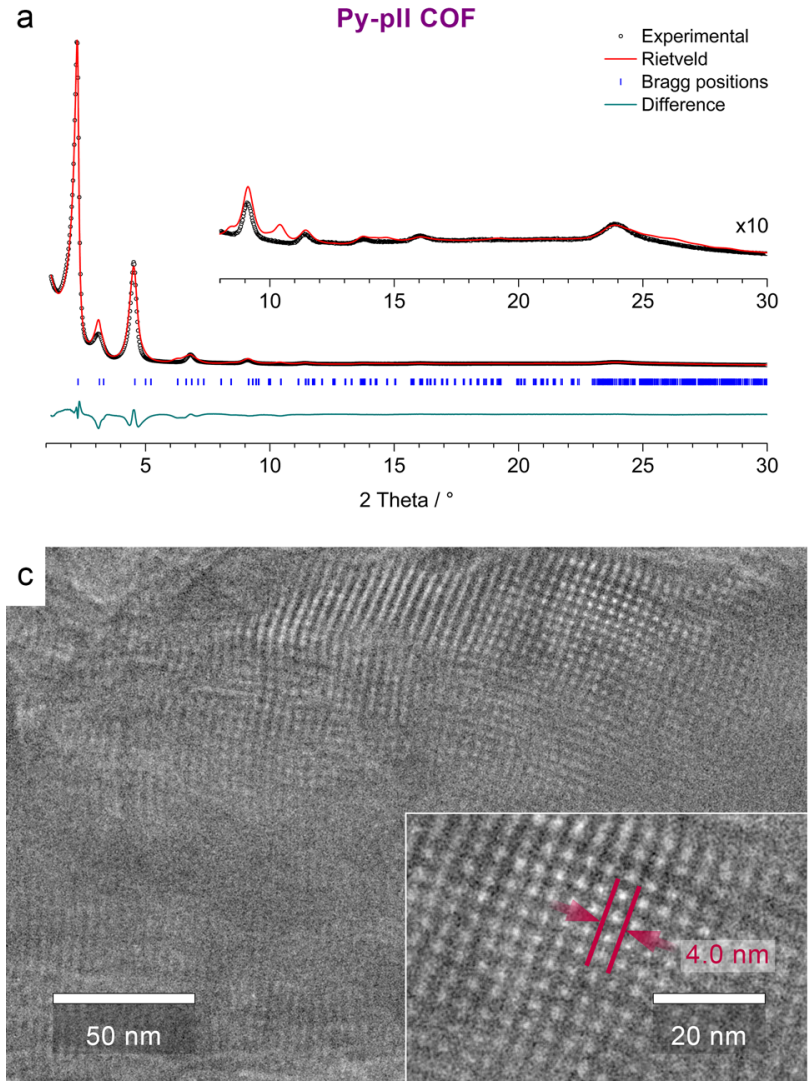

b

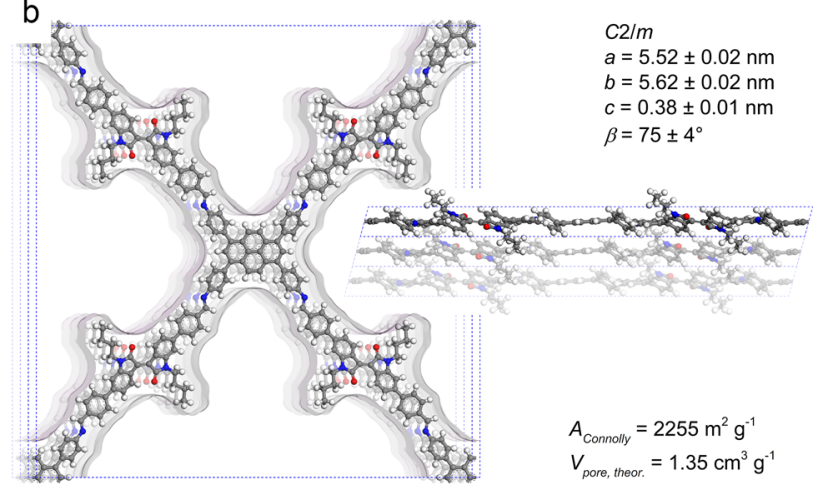

d

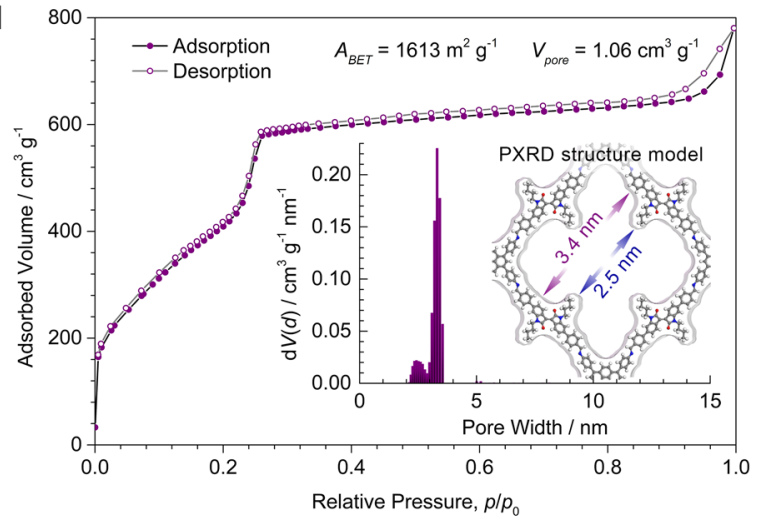

Figure 2. (a) Experimental PXRD pattern (black dots) of the Py-pII COF. Rietveld refinement (red line) using the C2/m-symmetric DFToptimized structure model shown in (b) provides a very good fit to the experimental data with only minimal differences (the green line shows the difference plot between the experimental and the Rietveld-refined PXRD patterns; $\left.R_{\mathrm{wp}}=7.86 \%, R_{\mathrm{p}}=13.9 \%\right)$. Bragg positions are indicated by blue ticks. Inset, magnified view of the $2 \theta>8^{\circ}$ region. (b) The Py-pII COF unit cell with the viewing direction normal to the $a-b$ plane (left) and along $b$ (right), and the Connolly surface calculated for a nitrogen-sized probe molecule. Crystallographic data are available as Supporting Information. (c) High-resolution TEM image of a polycrystalline Py-pII COF sample showing the pseudo-quadratic arrangement of the mesopores (top right), and the parallel alignment of the pore channels (top and bottom left). Inset, magnified view onto a COF domain showing the pseudo-quadratic geometry with a periodicity of $4.0 \pm 0.2 \mathrm{~nm}$. (d) Nitrogen sorption isotherm recorded at $77 \mathrm{~K}$. Insets, QSDFT calculation using an equilibrium model yields a very narrow pore size distribution centered at $3.3 \mathrm{~nm}$ with an additional porosity at around $2.5 \mathrm{~nm}$ due to the pII alkyl chains. These values agree very well with the wall-to-wall distance and the reduced pore diameter due to the alkyl chains derived from the Rietveld-refined structure model (purple and blue arrows).

COFs with significant $\pi$-overlap within the self-assembled columns of both subunits and excellent crystallinity. ${ }^{16,33,34}$

The imine-linked Py-pII COF was obtained via acidcatalyzed solvothermal synthesis as a dark purple powder (see the SI for experimental details).

Successful formation of a crystalline framework was confirmed by powder X-ray diffraction (PXRD; Figure 2a). The diffraction pattern exhibits a number of well-defined reflections and only weak background, highlighting the high degree of long-range order in this material. Rietveld refinement in the monoclinic space group $\mathrm{C} 2 / \mathrm{m}$ using a DFT optimized structure model reproduced the experimental pattern very well and yielded the lattice parameters $a=5.52 \pm 0.02 \mathrm{~nm}, b=5.62$ $\pm 0.02 \mathrm{~nm}, c=0.38 \pm 0.01 \mathrm{~nm}$, and $\beta=75 \pm 4^{\circ}$ (Figure 2b). Owing to the large number of light atoms in the unit cell and the peak broadening due to the inherent flexibility of the COF network it is not possible to refine the coordinates of individual atoms. We therefore observe some deviations in the intensities of higher-index reflections that are primarily attributed to slight differences between the DFT-optimized structure model and the actual COF structure.

High-resolution transmission electron microscopy (TEM) further confirms the formation of a periodic, porous framework
(Figure 2c). Domains that are oriented along the crystallographic $c$ axis show the pseudo-quadratic arrangement with a periodicity of $4.0 \pm 0.2 \mathrm{~nm}$, in excellent agreement with the inplane pyrene-to-pyrene distance of $3.93 \mathrm{~nm}$ in the Rietveldrefined COF structure.

The nitrogen sorption isotherm of the Py-pII COF exhibits a type IV isotherm shape with a sharp step around $p / p_{0}=0.24$ (Figure 2d). Pore size analysis by quenched solid density functional theory (QSDFT) using an equilibrium model for cylindrical pores reveals a distribution ranging from 2.1 to 3.4 $\mathrm{nm}$ with a main pore diameter of $3.3 \mathrm{~nm}$ and a second maximum at $2.5 \mathrm{~nm}$. Unlike most COFs reported to date, the Py-pII COF features butyl chains that extend into the pore, giving rise to a shamrock-shaped cross-section and resulting in more than one pore size. We notice that the maximum of the pore size distribution is in excellent agreement with the simulated wall-to-wall distance of $3.4 \mathrm{~nm}$, while the porosity at smaller pore diameter coincides with the reduced pore width of $2.5 \mathrm{~nm}$ due to the alkyl chains. ${ }^{35}$ The Brunauer-EmmettTeller (BET) surface area is $1613 \mathrm{~m}^{2} \mathrm{~g}^{-1}$ with a total pore volume of $1.06 \mathrm{~cm}^{3} \mathrm{~g}^{-1}$, in reasonable agreement with the Connolly surface of $2255 \mathrm{~m}^{2} \mathrm{~g}^{-1}$ and a theoretical pore volume of $1.35 \mathrm{~cm}^{3} \mathrm{~g}^{-1}$, confirming that the pores are open and 
a

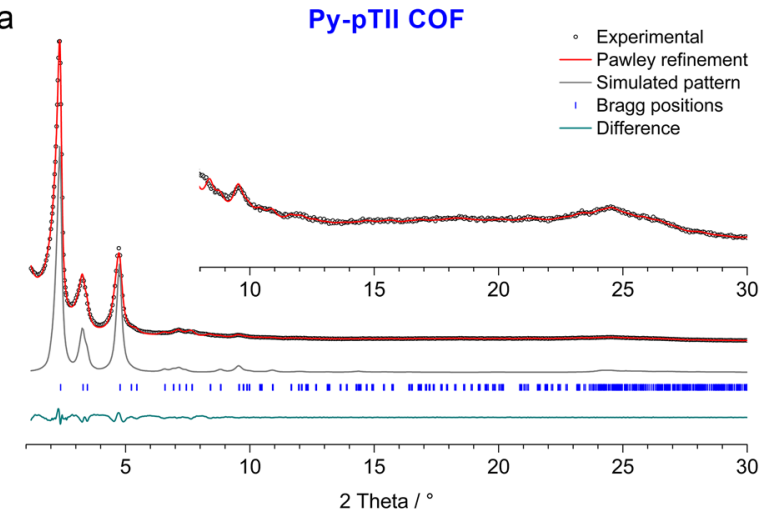

d

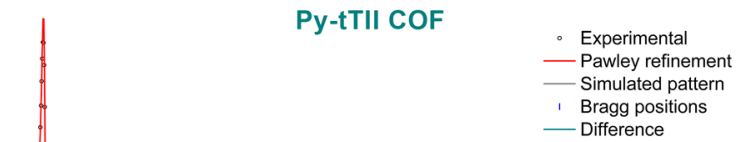

b

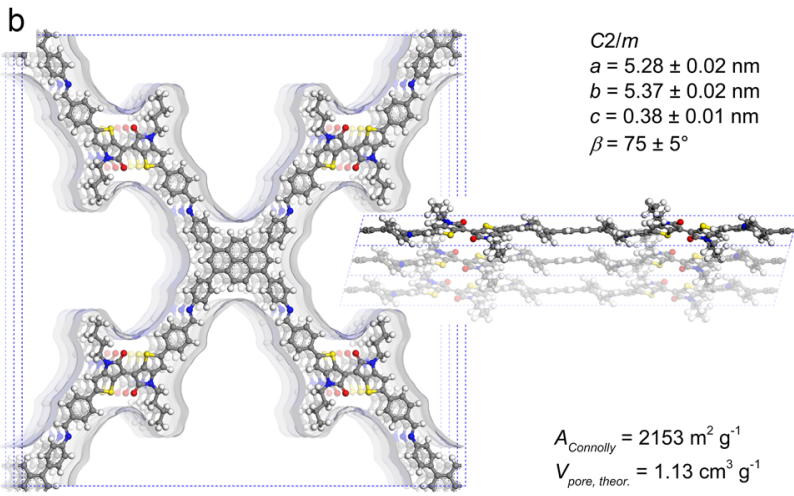

c

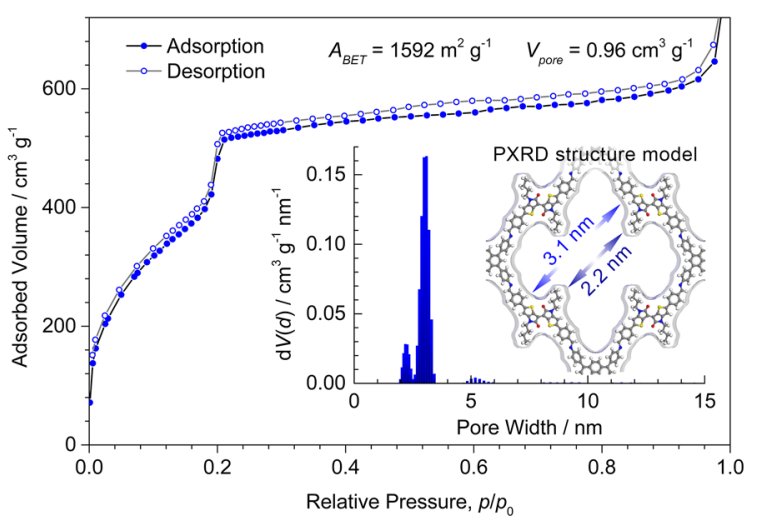

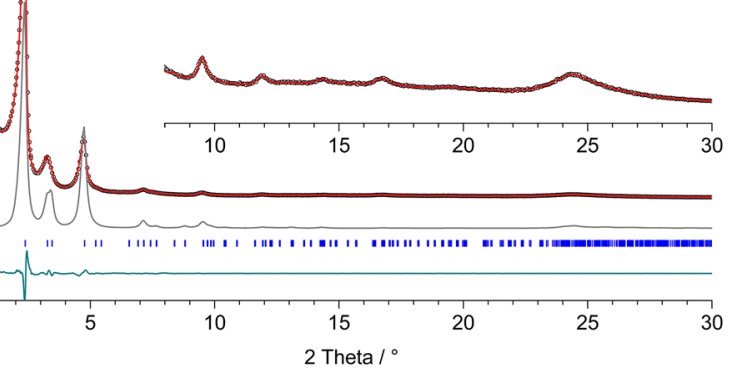

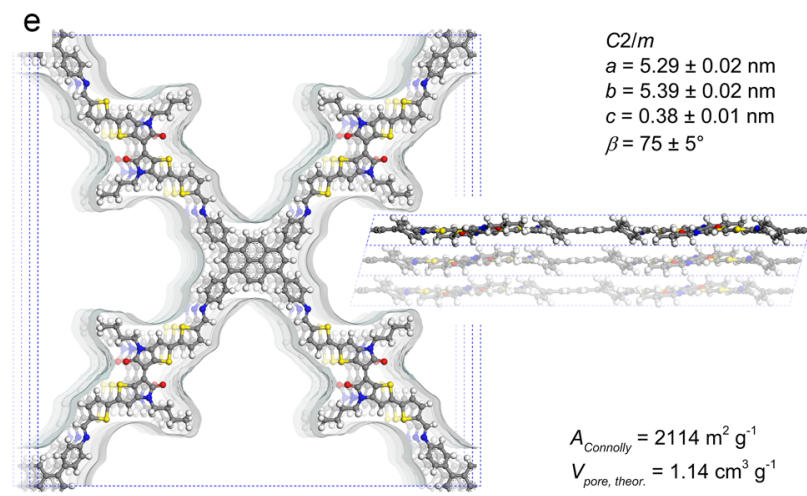

$f$

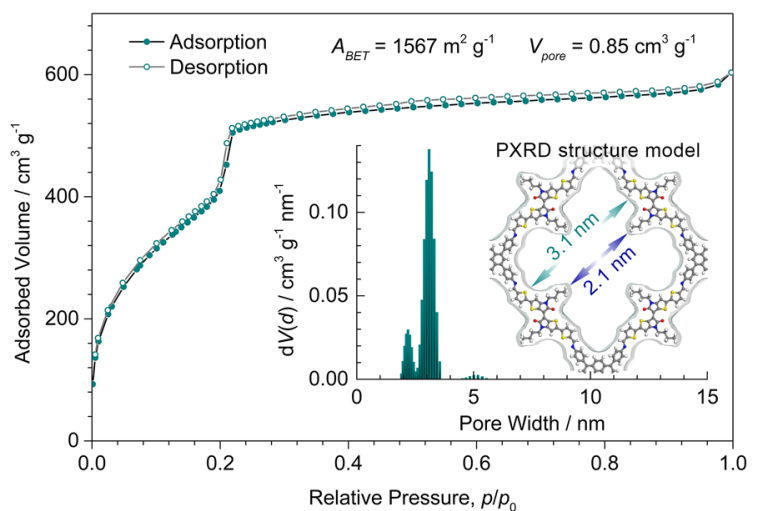

Figure 3. (a,d) Experimental PXRD patterns (black dots) of the thienoisoindigo-bridged Py-pTII and Py-tTII COFs, respectively. For both COFs, Pawley refinements (red lines) in the space group $\mathrm{C} 2 / \mathrm{m}$ provide an excellent fit to the experimental data. Insets, magnified view of the $2 \theta>8^{\circ}$ region. The simulated PXRD patterns (gray lines) based on the structure models shown in (b) and (e), respectively, agree very well with the experimental and refined patterns of the frameworks. Differences in the peak intensities might stem from slight differences between the simulations and the actual COF structures. (b,e) The Py-pTII and Py-tTII COF unit cells with the viewing direction normal to the $a-b$ plane (left) and along $b$ (right), and the Connolly surfaces calculated for a nitrogen-sized probe molecule. (c,f) Nitrogen sorption isotherms recorded at $77 \mathrm{~K}$. Insets, QSDFT calculations using an equilibrium model yield very narrow pore size distributions with maxima at 3.0 and $3.1 \mathrm{~nm}$, respectively, and an additional porosity at around $2.2 \mathrm{~nm}$ due to the alkyl chains of the thienoisoindigo moieties. These values agree very well with the wall-to-wall distances and the reduced pore diameters due to the alkyl chains derived from the refined structure models (blue and green arrows).

accessible. Due to the alkyl chains that split the pore into smaller compartments with highly concave surfaces, the Connolly surface tends to be systematically higher than the surface that can be occupied by nitrogen molecules during the sorption experiment. ${ }^{36}$

Recent studies on $N, N^{\prime}$-dimethyl-isoindigo showed that due to the steric repulsion between the protons of the benzene ring and the oxygen atoms of the ketopyrrole, the molecule crystallizes in a slightly twisted configuration with a rotation of the two oxindole rings along the central double bond. ${ }^{32}$ In a
COF, such deviation from a truly planar conformation might not only reduce the effective $\pi$-conjugation in the molecule, but could also affect the crystallinity and stability of the framework.

Attempting to overcome these potential structural drawbacks, thieno-modified versions of the isoindigo core have been developed. ${ }^{37}$ In thienoisoindigo (TII), the unfavorable repulsion between the ketones and the adjacent hydrogen atoms is replaced by a favorable electrostatic attraction between the sufficiently spaced ketones and sulfur atoms, thus rendering the molecule entirely planar. ${ }^{38}$ In order to test the influence of 

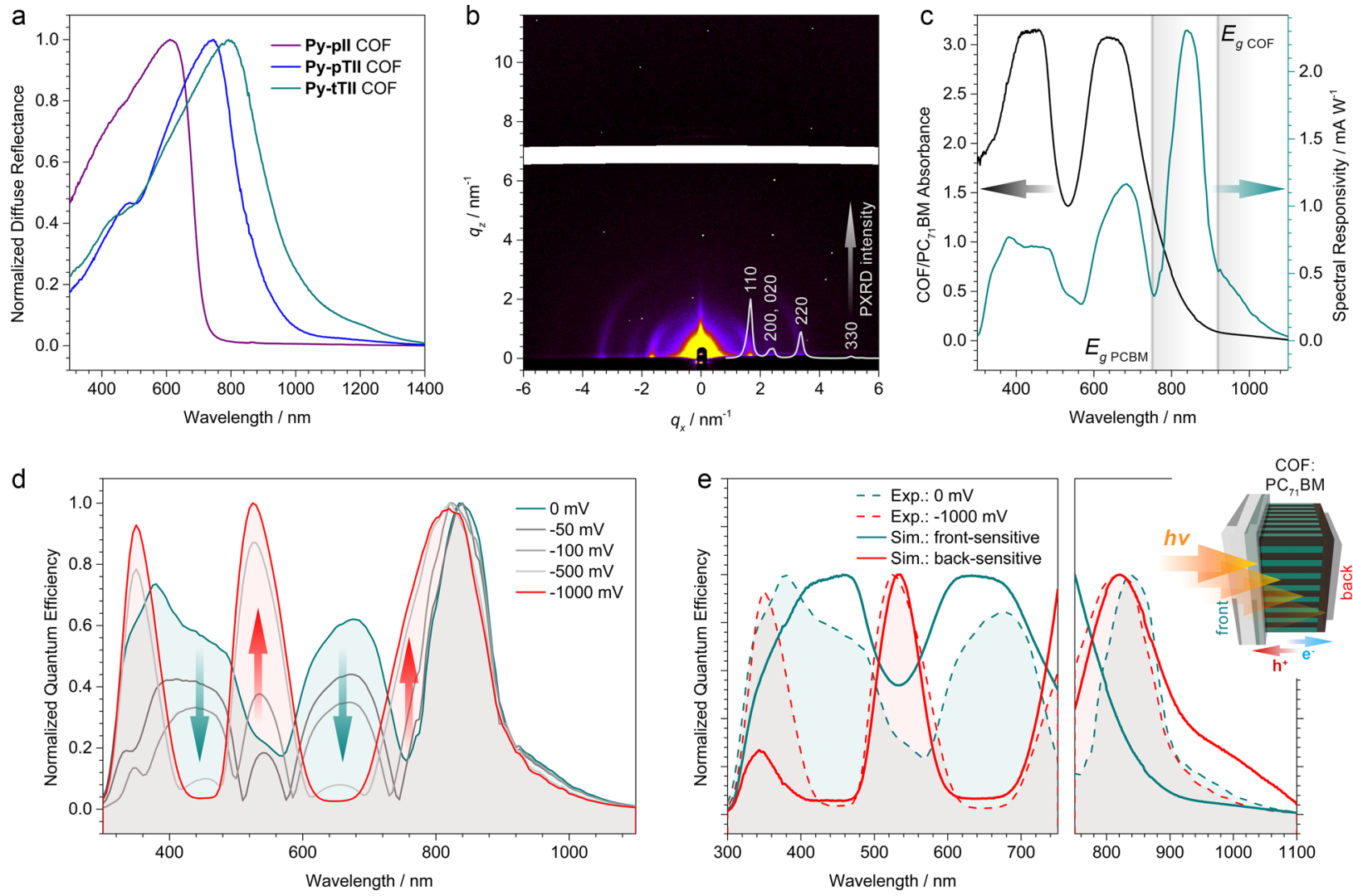

Figure 4. (a) UV-vis-NIR diffuse reflectance spectra of the Py-pII, Py-pTII, and Py-tTII COF powders dispersed in $\mathrm{BaSO}_{4}$. (b) GISAXS pattern of a Py-tTII COF film grown on ITO/ $\mathrm{MoO}_{x}$ indicating a predominant orientation of the COF domains with their $a-b$ plane parallel to the substrate. Inset, the simulated Py-tTII COF PXRD pattern. The reflections observed in the GISAXS pattern correspond to the 110, 020 and 200, 220, and 330 sets of lattice planes. (c) Spectral responsivity of the Py-tTII COF-based photodetector without external voltage bias (green) and the transmission absorbance of the COF:PC ${ }_{71} \mathrm{BM}$ active layer (black). Gray lines indicate the optical band gaps of $\mathrm{PC}_{71} \mathrm{BM}$ and the COF. (d) Upon application of an external voltage to the photodetector, the quantum efficiency in the green and NIR regions up to $750 \mathrm{~nm}$ is greatly enhanced. This is accompanied by a reduced sensitivity to blue and red light, eventually leading to an inversion of the sensitivity profile across the visible spectrum at $1000 \mathrm{mV}$ reverse bias. (e) A qualitative description of these characteristics can be derived from modeling the spectral distributions of collected charge carriers. While without voltage bias the device responds mostly to photons absorbed close to the front electrode (green line), the photoresponse at -1000 $\mathrm{mV}$ indicates a predominant sensitivity toward photons that penetrate deep into the active layer and are absorbed close to the back electrode (red line). Inset, illustration of the photodetector device layout.

this added planarity on the structure and electronic properties of our COFs, we constructed two TII-based building blocks (Figure 1a). According to our structure simulations, the 5,5'bis(4-formylphenyl)- $N, N^{\prime}$-dibutyl-thienoisoindigo (pTII) features a planar core that is flanked by slightly tilted phenyl rings (Figure 1c). Replacing the latter by thiophenes results in an entirely planar conformation of the 5,5'-bis (2-formylthiophen5-yl)-N,N'-dibutyl-thienoisoindigo (tTII) building block (Figure $1 \mathrm{~d})$.

The UV-vis spectra of pTII and tTII feature strong absorption bands in the blue and the red-to-NIR regions (SI, Figure S5a). While the $\pi-\pi^{*}$ transitions appear slightly blueshifted compared to the pII, the ICT bands of the TII building blocks are much stronger and considerably red-shifted to 480$730 \mathrm{~nm}$ and $500-750 \mathrm{~nm}$ for pTII and tTII, respectively. The corresponding optical band gaps, estimated from Tauc plots for direct allowed transitions, are 1.75 and $1.68 \mathrm{eV}$, respectively (SI, Figure S5c,d).

Acid-catalyzed co-condensation of Py with two equivalents of the TII building blocks yielded the Py-pTII and Py-tTII COFs as dark blue- and green-colored powders, respectively. Pawley refinement of the Py-pTII and Py-tTII COF PXRD patterns (Figures 3a, d), using the force-field-optimized structure models displayed in Figures $3 \mathrm{~b}$ and 3e, respectively, matched the experimental patterns very well. The refined unit cell parameters are $a=5.28 \pm 0.02 \mathrm{~nm}, b=5.37 \pm 0.02 \mathrm{~nm}, c=$ $0.38 \pm 0.01 \mathrm{~nm}$, and $\beta=75 \pm 5^{\circ}$ for the Py-pTII COF, and $a=$ $5.29 \pm 0.02 \mathrm{~nm}, b=5.39 \pm 0.02 \mathrm{~nm}, c=0.38 \pm 0.01 \mathrm{~nm}$, and $\beta$ $=75 \pm 5^{\circ}$ for the Py-tTII COF. In both COFs, the shorter length and slightly different geometry of the TII building blocks give rise to a more contracted framework compared to the $\mathbf{P y}$ pII COF.

The nitrogen sorption isotherms and corresponding QSDFT-derived pore size distributions are qualitatively similar to the Py-pII COF, but are, as anticipated from the refined COF structures, slightly shifted toward smaller pore diameters (Figures $3 \mathrm{c}, \mathrm{f}$ ). The pore size distribution exhibits again two maxima that stem from the shamrock-shaped pore crosssection. For both COFs, the main peak corresponds to the wallto-wall distance of $3.1 \mathrm{~nm}$, and the smaller one relates to the reduced pore diagonal due to the alkyl chains. The BET surface areas are $1592 \mathrm{~m}^{2} \mathrm{~g}^{-1}$ and $1567 \mathrm{~m}^{2} \mathrm{~g}^{-1}$ with total pore volumes of $0.96 \mathrm{~cm}^{3} \mathrm{~g}^{-1}$ and $0.85 \mathrm{~cm}^{3} \mathrm{~g}^{-1}$ for the Py-pTII and Py-tTII COFs, respectively. As discussed above for the Py-pII COF, differences between these values and the Connolly surface areas 
and theoretical pore volumes might arise from the pore geometry. $^{36}$

The integration of the isoindigo- and thienoisoindigocontaining building blocks produces strongly colored frameworks that absorb light across the visible and parts of the NIR spectrum (Figure 4a). The optical band gaps, estimated from the corresponding Tauc plots for direct allowed transitions, are 1.78, 1.48, and $1.36 \mathrm{eV}$, for the Py-pII, Py-pTII, and Py-tTII COFs, respectively (SI, Figure S6).

2D COFs possess very anisotropic electronic properties with the highest conductivity typically being along the $\pi$-stacked molecular columns. For application as an active component in a photodiode structure, these columns should therefore be aligned vertically to the substrate. The growth of oriented films on non-epitaxial substrates, during which the inherent anisotropy of the COF structure itself generates the preferred vertical orientation, has previously been achieved for boronate ester-linked COFs. ${ }^{19}$ We adapted this strategy for the growth of the imine-linked Py-tTII COF on $\mathrm{MoO}_{x}$-modified indium-tin oxide (ITO) transparent electrodes (see the SI for details).

Grazing-incidence small-angle X-ray scattering (GISAXS) measurements confirm that the resulting COF films are highly textured (Figure 4b). The intensity of the reflections corresponding to the 110, 020 and 200,220, and 330 sets of lattice planes is highest directly above the sample horizon, indicating that most COF domains are oriented with their $a-b$ plane parallel to the substrate (SI, Figure S7). Taking into account the monoclinic unit cell, the $\pi$-stacked columns thus extend away from the substrate at an angle of $\beta=75^{\circ}$. This orientation also ensures that the pores are open toward the film surface and can be used for the subsequent infiltration with a complementary semiconductor.

For the construction of a COF-based NIR photodetector, we infiltrated a $450 \mathrm{~nm}$ thick oriented Py-tTII COF film grown on a hole-selective ITO $/ \mathrm{MoO}_{x}$ electrode with $[6,6]$-phenyl $\mathrm{C}_{71}$ butyric acid methyl ester ( $\left.\mathrm{PC}_{71} \mathrm{BM}\right)$, thus forming an ordered interdigitated heterojunction. The device was completed by deposition of an electron-selective back contact consisting of poly $[(9,9-$ bis $(3-(N, N$-dimethylamino $)$ propyl)fluorene $)$-alt(9,9-dioctylfluorene)] (PFN) and Ag. While the degree of infiltration cannot be assessed via photoluminescence (PL) quenching $^{19,22}$ for this particular COF due to its very low PL quantum yield, comparing the $\mathrm{COF}: \mathrm{PC}_{71} \mathrm{BM}$ photodetection capabilities with a non-infiltrated COF-only device provides evidence for a predominant filling of the COF pores (SI, Figure S8d-f).

At short-circuit conditions the COF: $\mathrm{PC}_{71} \mathrm{BM}$ device exhibits a panchromatic photoresponse ranging from 300 to $1100 \mathrm{~nm}$ (Figure 4c, green line). For photon energies above the optical band gap of $\mathrm{PC}_{71} \mathrm{BM}$ (i.e., $\lambda<750 \mathrm{~nm}$ ), the spectral responsivity follows the absorbance of the COF:PC ${ }_{71} \mathrm{BM}$ heterojunction (Figure 4c, black line). The strong absorption and responsivity bands centered at 450 and $650 \mathrm{~nm}$, respectively, are mainly attributed to the $\pi-\pi^{*}$ transitions and the ICT band of tTII. At longer wavelengths, however, the photodetector exhibits an additional sensitivity peak around $840 \mathrm{~nm}$ that does not reflect an absorption feature of the COF: $\mathrm{PC}_{71} \mathrm{BM}$ heterojunction. This might be a first indication that the photophysics of the device change at photon energies at which only the COF can be photoexcited (see the discussion below).

In the absence of an external voltage bias, the peak responsivity is $2.3 \mathrm{~mA} \mathrm{~W}^{-1}$ at $840 \mathrm{~nm}$. When applying a reverse bias to aid the extraction of the photogenerated charge carriers, this can be increased to above $12 \mathrm{~mA} \mathrm{~W}^{-1}$ at -1000 $\mathrm{mV}$ (SI, Figure S8a).

Besides increasing responsivity and external quantum efficiency (EQE), applying a reverse bias has a profound effect on the photoresponse spectrum (Figure $4 \mathrm{~d}$ ). At $0 \mathrm{mV}$ bias, the photodetector is sensitive toward blue and red light, and relatively insensitive to green and NIR light shorter than 750 $\mathrm{nm}$. With increasing reverse bias, the relative and absolute sensitivities in the blue and red spectral region drop, while they improve in the green and NIR regions, ultimately inverting the sensitivity characteristics of the device throughout the 400-750 $\mathrm{nm}$ range. At longer wavelengths, where only the COF can be photoexcited, the shape of the EQE spectrum is hardly affected by applying a reverse bias.

In order to derive a qualitative description of these observations, we modeled the spectral distribution of the absorbed photons and hence the photogenerated charge carriers throughout the active layer (see the SI, section J for a detailed discussion). Considering the inevitable transport losses in thick organic bulk heterojunctions, the probability of a charge carrier pair to be collected at the respective electrodes, $\eta_{\text {coll }}$, depends on where in the active layer it has been generated. $\eta_{\text {coll }}$ is hereby limited by the carrier species (electron or hole) that has the shorter transport distance. Comparing the measured EQE spectra to the simulated spectral distributions of absorbed photons thus allows us to conclude from where inside the active layer the collected charge carriers originate. For the sake of simplicity, we focus our discussion on the two extreme cases of a front-sensitive $\left(\eta_{\text {coll }}\right.$ decreases with distance from front electrode, i.e., the illuminated side) and a backsensitive $\left(\eta_{\text {coll }}\right.$ increases toward back of the active layer) device (SI, Figure S9).

We first consider the $300-750 \mathrm{~nm}$ region, where both components of the heterojunction can be photoexcited. Without an external bias voltage, the observed EQE resembles the simulated photoresponse of a front-sensitive device, suggesting that the device performance is limited by the collection of holes at the front electrode (Figure 4e, green lines). If a reverse bias is applied, however, the EQE spectrum transforms into the photoresponse of a back-sensitive device, indicating that under these conditions the collection of electrons at the back electrode becomes the limiting factor (Figure $4 \mathrm{e}$, red lines). Here, the incident light has been attenuated by the optically thick COF:PC ${ }_{71} \mathrm{BM}$ film and hence the spectrum resembles the inverse of the absorption spectrum. ${ }^{39,40}$ This voltage-switchable spectral response is fully reversible (SI, Figure S8c).

In the longer-wavelength region, where only the COF can be photoexcited, the EQE spectrum exhibits a relatively sharp peak around $840 \mathrm{~nm}$. Optical modeling of the absorbed photons in this region (SI, Figure S9a) suggests that rather than corresponding to an absorption feature of the COF, this peak is caused by the balance between the penetration of light deep into the active layer (limiting factor at $750-840 \mathrm{~nm}$ ) and the absorption capabilities of the COF (dominant factor at 840$1100 \mathrm{~nm}$ ). Here, the device is back-sensitive, i.e. limited by the collection of electrons irrespective of the applied bias (Figure $4 \mathrm{e}$, right part).

A more detailed analysis of the origin of these device characteristics is beyond the scope of this work and will be the subject of future studies. 


\section{CONCLUSION}

In this work we have developed the first near-infraredabsorbing $2 \mathrm{D}$ covalent organic frameworks. The incorporation of donor-acceptor-type isoindigo- and thienoisoindigo-based building blocks leads to the formation of strongly light absorbing, stable and porous networks. Growing these materials as an oriented thin film allowed us to construct an interdigitated heterojunction upon infiltration of the COF pores with a soluble fullerene derivative. This heterojunction was successfully applied as the active layer in a UV- to NIRresponsive photodetector. We found that the spectral response of the device could be switched reversibly from blue- and redsensitive to green- and NIR-sensitive by changing the bias voltage. To the best of our knowledge, this is the first time that such nearly complete inversion of spectral sensitivity has been observed. This effect could lead to potential applications in information technology or spectral imaging. Future work will be dedicated to developing a more quantitative understanding of the COF photophysics.

\section{ASSOCIATED CONTENT}

\section{S Supporting Information}

The Supporting Information is available free of charge on the ACS Publications website at DOI: 10.1021/jacs.7b06599.

Experimental methods, synthetic procedures, optical modeling, and additional spectroscopic data (PDF) X-ray crystallographic data for Py-pII COF (CIF)

\section{AUTHOR INFORMATION}

\section{Corresponding Authors}

*fa355@cam.ac.uk

*bein@lmu.de

\section{ORCID}

Florian Auras: 0000-0003-1709-4384

\section{Notes}

The authors declare no competing financial interest.

\section{ACKNOWLEDGMENTS}

The authors are grateful for funding from the German Science Foundation (DFG; Research Cluster NIM) and the Free State of Bavaria (Research Network SolTech). The research leading to these results received funding from the European Research Council under the European Union's Seventh Framework Programme (FP7/2007-2013)/ERC Grant Agreement No. 321339. The authors thank Dr. Markus Döblinger for the transmission electron microscopy.

\section{REFERENCES}

(1) Côte, A. P.; Benin, A. I.; Ockwig, N. W.; O’Keeffe, M.; Matzger, A. J.; Yaghi, O. M. Science 2005, 310, 1166.

(2) Uribe-Romo, F. J.; Hunt, J. R.; Furukawa, H.; Klöck, C.; O’Keeffe, M.; Yaghi, O. M. J. Am. Chem. Soc. 2009, 131, 4570.

(3) Beaudoin, D.; Maris, T.; Wuest, J. D. Nat. Chem. 2013, 5, 830.

(4) Spitler, E. L.; Dichtel, W. R. Nat. Chem. 2010, 2, 672.

(5) Duhović, S.; Dincă, M. Chem. Mater. 2015, 27, 5487.

(6) Huang, N.; Ding, X.; Kim, J.; Ihee, H.; Jiang, D. Angew. Chem., Int. Ed. 2015, 54, 8704.

(7) Fang, Q.; Zhuang, Z.; Gu, S.; Kaspar, R. B.; Zheng, J.; Wang, J.; Qiu, S.; Yan, Y. Nat. Commun. 2014, 5, 4503.

(8) Rabbani, M. G.; Sekizkardes, A. K.; Kahveci, Z.; Reich, T. E.; Ding, R.; El-Kaderi, H. M. Chem. - Eur. J. 2013, 19, 3324.
(9) Calik, M.; Sick, T.; Dogru, M.; Döblinger, M.; Datz, S.; Budde, H.; Hartschuh, A.; Auras, F.; Bein, T. J. Am. Chem. Soc. 2016, 138, 1234.

(10) Lin, S.; Diercks, C. S.; Zhang, Y.-B.; Kornienko, N.; Nichols, E. M.; Zhao, Y.; Paris, A. R.; Kim, D.; Yang, P.; Yaghi, O. M.; Chang, C. J. Science 2015, 349, 1208.

(11) Furukawa, H.; Yaghi, O. M. J. Am. Chem. Soc. 2009, 131, 8875.

(12) Shinde, D. B.; Kandambeth, S.; Pachfule, P.; Kumar, R. R.; Banerjee, R. Chem. Commun. 2015, 51, 310.

(13) Vyas, V. S.; Haase, F.; Stegbauer, L.; Savasci, G.; Podjaski, F.; Ochsenfeld, C.; Lotsch, B. V. Nat. Commun. 2015, 6, 8508.

(14) Bertrand, G. H. V.; Michaelis, V. K.; Ong, T.-C.; Griffin, R. G.; Dinca, M. Proc. Natl. Acad. Sci. U. S. A. 2013, 110, 4923.

(15) Chen, L.; Furukawa, K.; Gao, J.; Nagai, A.; Nakamura, T.; Dong, Y.; Jiang, D. J. Am. Chem. Soc. 2014, 136, 9806.

(16) Keller, N.; Bessinger, D.; Reuter, S.; Calik, M.; Ascherl, L.; Hanusch, F. C.; Auras, F.; Bein, T. J. Am. Chem. Soc. 2017, 139, 8194.

(17) Auras, F.; Ascherl, L.; Hakimioun, A. H.; Margraf, J. T.; Hanusch, F. C.; Reuter, S.; Bessinger, D.; Döblinger, M.; Hettstedt, C.; Karaghiosoff, K.; Herbert, S.; Knochel, P.; Clark, T.; Bein, T. J. Am. Chem. Soc. 2016, 138, 16703.

(18) Ascherl, L.; Sick, T.; Margraf, J. T.; Lapidus, S. H.; Calik, M.; Hettstedt, C.; Karaghiosoff, K.; Döblinger, M.; Clark, T.; Chapman, K. W.; Auras, F.; Bein, T. Nat. Chem. 2016, 8, 310.

(19) Medina, D. D.; Werner, V.; Auras, F.; Tautz, R.; Dogru, M.; Schuster, J.; Linke, S.; Döblinger, M.; Feldmann, J.; Knochel, P.; Bein, T. ACS Nano 2014, 8, 4042.

(20) Jin, S.; Supur, M.; Addicoat, M.; Furukawa, K.; Chen, L.; Nakamura, T.; Fukuzumi, S.; Irle, S.; Jiang, D. J. Am. Chem. Soc. 2015 137, 7817.

(21) Medina, D. D.; Petrus, M. L.; Jumabekov, A. N.; Margraf, J. T.; Weinberger, S.; Rotter, J. M.; Clark, T.; Bein, T. ACS Nano 2017, 11, 2706.

(22) Dogru, M.; Handloser, M.; Auras, F.; Kunz, T.; Medina, D.; Hartschuh, A.; Knochel, P.; Bein, T. Angew. Chem., Int. Ed. 2013, 52, 2920.

(23) Calik, M.; Auras, F.; Salonen, L. M.; Bader, K.; Grill, I.; Handloser, M.; Medina, D. D.; Dogru, M.; Löbermann, F.; Trauner, D.; Hartschuh, A.; Bein, T. J. Am. Chem. Soc. 2014, 136, 17802.

(24) Guo, J.; Xu, Y.; Jin, S.; Chen, L.; Kaji, T.; Honsho, Y.; Addicoat, M. A.; Kim, J.; Saeki, A.; Ihee, H.; Seki, S.; Irle, S.; Hiramoto, M.; Jiang, D. Nat. Commun. 2013, 4, 2736.

(25) Peet, J.; Kim, J. Y.; Coates, N. E.; Ma, W. L.; Moses, D.; Heeger, A. J.; Bazan, G. C. Nat. Mater. 2007, 6, 497.

(26) Chen, M. S.; Niskala, J. R.; Unruh, D. A.; Chu, C. K.; Lee, O. P.; Fréchet, J. M. J. Chem. Mater. 2013, 25, 4088.

(27) Yue, W.; Ashraf, R. S.; Nielsen, C. B.; Collado-Fregoso, E.; Niazi, M. R.; Yousaf, S. A.; Kirkus, M.; Chen, H.-Y.; Amassian, A.; Durrant, J. R.; McCulloch, I. Adv. Mater. 2015, 27, 4702.

(28) Kim, G.; Kang, S.-J.; Dutta, G. K.; Han, Y.-K.; Shin, T. J.; Noh, Y.-Y.; Yang, C. J. Am. Chem. Soc. 2014, 136, 9477.

(29) Meager, I.; Nikolka, M.; Schroeder, B. C.; Nielsen, C. B.; Planells, M.; Bronstein, H.; Rumer, J. W.; James, D. I.; Ashraf, R. S.; Sadhanala, A.; Hayoz, P.; Flores, J.-C.; Sirringhaus, H.; McCulloch, I. Adv. Funct. Mater. 2014, 24, 7109.

(30) Matsumoto, M.; Dasari, R. R.; Ji, W.; Feriante, C. H.; Parker, T. C.; Marder, S. R.; Dichtel, W. R. J. Am. Chem. Soc. 2017, 139, 4999.

(31) Stalder, R.; Mei, J.; Reynolds, J. R. Macromolecules 2010, 43, 8348.

(32) Voronina, Y. K.; Krivolapov, D. B.; Bogdanov, A. V.; Mironov, V. F.; Litvinov, I. A. J. Struct. Chem. 2012, 53, 413.

(33) Chen, X.; Huang, N.; Gao, J.; Xu, H.; Xu, F.; Jiang, D. Chem. Commun. 2014, 50, 6161.

(34) Wu, Y.; Xu, H.; Chen, X.; Gao, J.; Jiang, D. Chem. Commun. 2015, 51, 10096.

(35) Lohse, M. S.; Rotter, J. M.; Margraf, J. T.; Werner, V.; Becker, M.; Herbert, S.; Knochel, P.; Clark, T.; Bein, T.; Medina, D. D. CrystEngComm 2016, 18, 4295. 
(36) Düren, T.; Millange, F.; Férey, G.; Walton, K. S.; Snurr, R. Q. J. Phys. Chem. C 2007, 111, 15350.

(37) Van Pruissen, G. W. P.; Gholamrezaie, F.; Wienk, M. M.; Janssen, R. A. J. J. Mater. Chem. 2012, 22, 20387.

(38) Dutta, G. K.; Han, A.-R.; Lee, J.; Kim, Y.; Oh, J. H.; Yang, C. Adv. Funct. Mater. 2013, 23, 5317.

(39) Li, Y.; Auras, F.; Löbermann, F.; Döblinger, M.; Schuster, J.; Peter, L.; Trauner, D.; Bein, T. J. Am. Chem. Soc. 2013, 135, 18513.

(40) Kubo, M.; Kaji, T.; Hiramoto, M. AIP Adv. 2011, 1, 032177. 